\title{
Etiology. Epidemiology, Diagnosis and Current Therapeutic Protocols for Parkinson's Disease (PD): An Overview
}

\author{
Mimansha Pradahan ${ }^{1}$, Rahul Srivastava ${ }^{2}$ \\ ${ }^{1}$ Intern, Rama Dental College, Hospital \& Research Centre, Kanpur (U.P), ${ }^{2}$ Reader, Department of Oral Medicine \& Radiology, \\ Rama Dental College, Hospital \& Research Centre, Kanpur (U.P), India
}

Corresponding author: Dr. Rahul Srivastava, Reader, Department of Oral Medicine \& Radiology, Rama Dental College, Hospital \& Research Centre, Kanpur (U.P), India

DOI: http://dx.doi.org/10.21276/ijcmsr.2020.5.1.41

(c) BY-NC-ND

How to cite this article: Mimansha Pradahan, Rahul Srivastava. Etiology. epidemiology, diagnosis and current therapeutic protocols for parkinson's disease (PD): an overview. International Journal of Contemporary Medicine Surgery and Radiology. 2020;5(1):A186-A191.

\section{A B S T R A C T}

Parkinson's disease (PD) is a neurodegenerative disorder that affects predominately dopamine-producing ("dopaminergic") neurons in a specific area of the brain called substantia nigra. PD is a progressive movement disorder with an increased mortality compared to the general population. Given a large number of motor and non-motor symptoms in patients with PD, the diagnosis is clinical and sometimes difficult. This review highlights the etiopathogenesis, clinical features, diagnosis and recent treatment modalities for Parkinson's disease.

Keywords: Parkinson's Disease, Motor Symptoms, PD Biomarkers, Deep Brain Stimulation.

\section{INTRODUCTION}

Dr. James Parkinson first described Parkinson's disease (PD) as a "shaking palsy" in 1817. After Alzheimer's disease, Parkinson's disease is the second most common neurodegenerative disorder. It is a chronic, progressive neurodegenerative disease with both motor and non-motor characteristics. The term parkinsonism is a complex of symptoms used to describe PD's motor features, including resting tremor, bradykinesia, and muscular rigidity. ${ }^{1,2}$ The Parkinson's Disease Foundation estimates that approximately 1 million Americans are currently suffering from the disease. The prevalence of PD in the U.S. is about 20 cases per 100,000 individuals per year (60,000 per year), with an average starting age of about 60 years. ${ }^{3}$ In 2006 Lau and Breteler reported that an estimated 10 million people in the world (i.e., approximately $0.3 \%$ of the world population) and $1 \%$ of those above 60 years are found to be affected with PD. ${ }^{4}$ The exact incidence and prevalence of PD in India is determined by very few population-based studies. The prevalence rate of Parkinsonism was found to be 33 per 100,000 (crude prevalence) and 76 per 100,000 (age adjusted) in a door-to-door survey conducted in Bangalore district in South Karnataka, India in 2004. ${ }^{5}$ The prevalence of Parkinsonism was found to be 45.82 per 100,000 from a study done by Das SK et al in Kolkata in 2006.6 According to Razdan $\mathrm{S}$ et al the prevalence was 14.1 per 100,000 in the state of Kashmir, whereas the age-adjusted prevalence was 134 per 100,000. ${ }^{7}$ A survey conducted by Bharucha NE et al in Mumbai's Parsi community, a small prosperous group, showed 192 per 100,000 prevalence, which was higher than the rest of the population. ${ }^{8}$ Parkinsonism had a very high prevalence of 17.6 percent (109/612 residents) in a surveillance in old-age homes in Bangalore. ${ }^{9}$

Research recommends that the pathophysiological changes related with PD may begin earlier than the onset of motor features and may include a number of nonmotor presentations, such as sleep disorders, depression, and cognitive changes. PD's variable but pronounced development has considerable impact on patients, families and society. Advanced and endstage illnesses can lead to excessive complications including death-related pneumonia. ${ }^{10}$ This review presents current strategies and recommendations for treating motor and non-motor symptoms in the different stages of Parkinson's disease.

\section{Pathophysiology}

Although it is primarily an elderly disease, individuals in their 30s and 40s have developed PD. PD is associated with multiple risk factors and genetic mutations. Risk factors for the illness include oxidative stress, free radical development, and a variety of environmental toxins. Risk factors associated with parkinson's disease are: ${ }^{11,12}$

1. Elevated cholesterol.

2. Environmental toxins like:

A. Carbon disulfide.

B. Cyanide.

C. Herbicides.

D. Methanol and organic solvents.

E Pesticides.

3. Head trauma.

4. High caloric intake.

5. Increased body mass index.

6. Inflammation associated with activation of microglia. 
7. Methcathinone (manganese content).

8. Methamphetamine/amphetamine abuse.

9. Mitochondrial dysfunction.

10. Nitric oxide toxicity.

11. Oxidative stress:

A. Formation of free radicals (e.g., hydrogen peroxide).

B. Potent neurotoxins (e.g., 1-methyl-4-phenyl1,2,3,6-tetrahydropyridine).

12. Post-infection states.

13. Signal-mediated apoptosis.

Gene Mutations

Gene mutations associated with Parkinson's Disease are:

1. Alpha-synuclein gene (SNCA).

2. Eukaryotic translation initiation factor 4 gamma 1 gene (EIF4G1).

3. Glucocerebrosidase gene (GBA).

4. Leucine-rich repeat kinase 2 (LRRK2) gene loci.

5. PTEN-induced putative kinase 1 (PINK1) gene loci.

6. Superoxide dismutase 2 gene (SOD2).

7. Vacuolar protein sorting 35 homolog gene (VPS35).

Parkinson's disease is a neurodegenerative syndrome with numerous neuronal motor and non-motor circuits.

There are two main pathological mechanisms characterizing it: (a) premature selective loss of dopamine neurons. (b) Accumulation of $\alpha$-synuclein Lewy bodies that are misfolded and accumulate in multiple systems in Parkinson's disease patients. Which phase takes place first is unclear. There is a progressive degeneration of neurons over many years based on clinical studies, with each affected site leading to specific symptoms of Parkinson's disease. ${ }^{13}$ Break staging of Lewy body deposition showed in Table 1

\section{Structural brain lesions}

Cerebrovascular disease, infectious brain lesions (e.g., cryptococcosis, neurosyphilis), postencephalitic (e.g., encephalitis lethargica), postanoxic injury, posttraumatic (e.g., dementia pugilistica), toxic (e.g., MPTP, ephedrone, manganese, carbon monoxide, cyanide), metabolic disorders (Wilson, Niemann-Pick Type C, Gaucher, GM1 gangliosidosis, phenylketonuria, cerebrotendinous xanthomatosis, maple syrup urine disease, mitochondrial disorders with striatal necrosis, ceroid neuronal lipofuscinosis), secondary Fahr's syndrome, non-Wilsonian acquired hepatocerebral degeneration, hemiparkinsonismhemiatrophy syndrome, hydrocephalus, and intracranial tumors.

\section{Drug-induced.}

Typical antipsychotics (e.g., haloperidol, chlorpromazine), most atypical antipsychotics (riperidone, olanzapine), tetrabenazine, reserpine, methyldopa, metoclopramide, flunarizine, cinnarizine, verapamil, valproic acid, lithium, and others. ${ }^{14}$

\section{Clinical Features \\ Motor symptoms}

From the motor point of view, PD is characterized by a clinical syndrome commonly known as parkinsonism, which involves four cardinal features: bradykinesia, rest tremor, rigidity and loss of posture and gait.

Bradykinesia: Refers to slow movements with a gradual loss of amplitude or speed during attempted rapid alternation of body segment movements. Clinically, bradykinesia can be tested by asking the patient to perform as rapidly and frequently as possible repetitive movements such as opening and closing the hand, tapping thumb and index fingers, or tapping the foot on the ground. The investigator will pay attention to the appearance of progressive slowness and/ or lack of amplitude, which could potentially lead to full arrest of the movement. Some clinical manifestations of bradykinesia include hypomimia (decreased facial expression and eye blinking, in milder stages called the "poker face"), hypophonia (softer voice), micrographia (progressively smaller handwriting), and difficulty swallowing.

Rest tremor (parkinsonian tremor): Is a rhythmic oscillating involuntary movement that occurs when the affected part of the body is relaxed and supported by a surface, thus eliminating the action of gravitational forces. In PD, the frequency of the rest tremor typically varies from low to medium $(3-6 \mathrm{~Hz})$, while the amplitude is quite variable, between less than 1 $\mathrm{cm}$ and $>10 \mathrm{~cm}$ wide. The most distinguishing tremor in this condition is the so-called "pill-rolling" type, a visual depiction resulting from the simultaneous rubbing of thumb and index finger movements against each other.

Rigidity: Refers to an increased muscle tone felt by passive movement of the affected segment (limbs or neck) during the examination, affecting both flexor and extensor muscle groups. This resistance is felt throughout the entire range of motion and does not increase with higher mobilization speed, which separates it from spasticity because of neuronal upper motor lesions. The classic "cog-wheel rigidity" can be felt during passive limb mobilization, particularly in the wrist, when resting tremor coexists.

\section{Postural and gait impairment:}

Due to the loss of postural reflexes, Parkinsonian patients tend to adopt a stooped posture which is a major contributor to falls. The Parkinsonian gait is slow, occurs on a narrow base and is characterized by short shuffling steps which give the observer the impression that the patient is chasing his own center of gravity. There is decreased arm swing, turning is slow and performed with multiple small steps, whereas gait freezing can occur particularly in crowded or narrow

\begin{tabular}{|l|l|l|}
\hline Stage & Sites affected by lewy bodies & Major symptoms \\
\hline $\mathrm{I}$ & Dorsal motor nucleus of the vagus nerve and olfactory tract & Constipation, anosmia \\
\hline $\mathrm{II}$ & Locus coeruleus and subcoeruleus complex & Sleep and mood dysfunction \\
\hline $\mathrm{III}$ & Substantia nigra & Motor symptoms of Parkinson disease \\
\hline $\mathrm{IV}-\mathrm{VI}$ & Cortical involvement & Dementia, psychosis \\
\hline \multicolumn{2}{|c|}{ Table-1: Break staging of Lewy body } \\
\hline
\end{tabular}


spots. The "pull test" is done to assess postural stability; the examiner stands behind the supine patient who has previously been warned of the "pull" applied to his / her shoulders and then encourages him / her to step back in order to regain equilibrium-some patients will fall without any postural reaction. ${ }^{14}$

1. Nonmotor symptoms ${ }^{15,16}$

- Neuropsychiatric features

- Apathy.

- Anxiety, panic attacks.

- Mood disorders, especially depression.

- Hallucinations, illusions, delusions.

- Cognitive deterioration, ranging from mild impairment to dementia.

2. Dysautonomia

- Orthostatic hypotension.

- Constipation.

- Urinary dysfunction (urgency, retention).

- Sexual dysfunction.

- Excessive sweating.

- Seborrhea.

- Sialorrhea (i.e., drooling, also attributable to decreased swallowing movements).

\section{Sleep disorders}

- Insomnia.

- Rapid eye movement (REM) behavior disorder.

- Restless legs syndrome.

- Periodic limb movements in sleep.

- Excessive daytime sleepiness.

\section{Sensory dysfunction}

- Hyposmia (i.e., loss of sense of smell).

- Decreased visual contrast and color discrimination.

- Decreased visual motion perception.

- Abnormal sensations, such as paresthesias (i.e., tingling).

- Pain.

- Fatigue.

\section{Orofacial manifestations ${ }^{17}$}

- Dental Caries and Periodontal Disease.

- Sialorrhea and Drooling.

- Xerostomia.

- Burning Mouth Syndrome.

- Mastication Disorders.

- Bruxism.

- $\quad$ Subjective Taste Impairment.

- "Masklike" facial appearance with infrequent blinking and lack of expression is caused by bradykinesis.

\section{DIAGNOSIS OF PARKINSON DISEASE}

The diagnosis of Parkinson's disease is usually based on clinical symptoms, history and evaluation and, over time, on the reaction to dopamine agents and the development of motor fluctuations. Motor symptoms of the condition begin asymmetrically and typically include a resting tremor, a soft voice (hypophonia), masked facies (initially identified as a reduced blink rate), small handwriting (micrographia), stiffness (rigidity), slowness of movements (bradykinesia), shuffling steps and difficulties with balance. ${ }^{18}$ The most widely accepted clinical criteria for the diagnosis of $\mathrm{PD}$ are those introduced by the UK PDS Brain Bank Criteria:

\section{Step 1. Diagnosis of a parkinsonian syndrome ${ }^{19}$}

Bradykinesia and at least one of the following:

- Muscular rigidity.

- Rest tremor (4-6 Hz).

- Postural instability unrelated to primary visual, cerebellar, vestibular or proprioceptive dysfunction.

\section{Step 2. Exclusion criteria for PD}

History of:

- Repeated strokes with stepwise progression

- Repeated head injury

- Antipsychotic or dopamine-depleting drugs

- Definite encephalitis and/or oculogyric crises on no drug treatment

- More than one affected relative

- Sustained remission

- Negative response to large doses of levodopa (if malabsorption excluded)

- Strictly unilateral features after 3 years

- Other neurological features: supranuclear gaze palsy, cerebellar signs, early severe autonomic involvement, Babinski sign, early severe dementia with disturbances of language, memory or praxis

- Exposure to known neurotoxin

- Presence of cerebral tumour or communicating hydrocephalus on neuroimaging.

\section{Step 3. Supportive criteria for PD}

Three or more required for diagnosis of definite PD:

- Unilateral onset

- Rest tremor present

- Progressive disorder

- Persistent asymmetry affecting the side of onset most

- Excellent response to levodopa

- Severe levodopa-induced chorea

- Levodopa response for over 5 years

- Clinical course of over 10 years.

\section{Neurochemical Biomarkers}

1. Orexin

Orexin is a neuropeptide hormone that is expressed by a small number of dorsolateral hypothalamus neurons, also known as hypocretin. The lateral and posterior neurons of the hypothalamus secrete Orexin. Orexin I concentration in PD patients is lower than in healthy individuals, and orexin level is correlated with disease severity. The more serious the disease is, the greater the loss of hypocretin neurons and the lower levels of orexin in CSF are observed..$^{20}$

\section{8-Hydroxy-2'-Deoxyguanosine}

Reactive oxygen species (ROS) species (such as O2-, $\mathrm{H} 2 \mathrm{O} 2$, and .OH) may cause damage to biological molecules through irreversible reactions that cause degenerative agingrelated processes. One of the ROS-caused DNA lesions is an oxidized form of 8-hydroxyguanine (8-OHG) known as 8-OHdG which can be used as a DNA damage biomarker. For PD patients a rise for $8-\mathrm{OHdG}$ serum levels was also assessed compared to normal individuals. ${ }^{21}$ 


\section{Peripheral Proteasomes and Caspase Activity}

Proteasomes are large protein complexes responsible for the degradation and removal of unwanted and misfolded proteins, and are therefore necessary for the survival of cells. In $\mathrm{PD}$ the accumulation of proteins within the neurons leads to the formation of intracellular pathological inclusions called LBs. Proteasome dysfunction can involve and associate with LBs in the formation of protein aggregates. Disturbing proteasome activity in PD mutations may result in accumulation of aggregated $\alpha$-syn. In some cases of PD, mitochondrial deficiency leads to increased ROS production and higher $\alpha$-syn oxidation resulting in increased ATP-independent proteasomal activity and higher $\alpha$-syn oligomerisation. In this case, depletion of ATP levels inhibits $26 \mathrm{~S}$ proteasome, but $20 \mathrm{~S}$ complex remains active and degrades the oxidised $\alpha$-syn. In advanced PD the severity and duration of PD correlate with reduced activity of $20 \mathrm{~S}$ proteasome and increased activity of caspase 3. Caspase activation and thus the initiation of apoptosis is the main reason for the reduction of proteasome $20 \mathrm{~S}$ activity. ${ }^{22,23}$

\section{Dopamine, Dopamine Receptor, and Dopamine Transporter Activity}

In the brain, dopamine is used as the noradrenaline (norepinephrine) and adrenaline (epinephrine) precursors. Loss of dopaminergic neurons in the midbrain and SN of PD brains leads to decrease in dopamine levels. The dopamine transporter (DAT) controls levels of dopamine by facilitating its recovery back to the cytosol. Free dopamine is toxic to neurons, however, since its oxidation produces poisonous reactive quinones. Hence the monoamine vesicular transporter 2 (VMAT2) stores excess dopamine in vesicles, some shift in the dopamine or DAT levels can therefore be an indication of PD..$^{24}$

\section{5. $\alpha$-Synuclein}

$\alpha$-Synuclein, found in aggregate and fibrillary form, has attracted considerable attention as a potential molecular biomarker of PD. Human $\alpha$-syn is predominantly expressed in neocortex, hippocampus, $\mathrm{SN}$, thalamus and cerebellum in the brain and is found in LBs. Changes in $\alpha$-syn levels have been reported in PD patients with CSF and plasma as compared to individuals with control. ${ }^{25}$

\section{Apolipoprotein A1 (ApoA1)}

ApoA1 is an apolipoprotein of $28 \mathrm{AA}$ with a molecular weight of about $28 \mathrm{kDa}$ that is the main component of HDL particles. ApoA1 and another apolipoprotein are responsible for carrying lipids in the brain. The CSF of PD patients reports lower levels of one isoform of apoA1 and tetranectin, suggesting that apoA1 is a potential biomarker for $\mathrm{PD}$, in particular due to their presence in CSF and peripheral circulation both free and in exosomes. ${ }^{26}$

\section{RNA-Based PD Biomarkers}

Recent investigation of microRNAs (miRNAs) in PD point to their emerging role as potential PD biomarkers, especially due to their presence in CSF and peripheral circulation both free and in exosomes. miRNAs are small, 21-24 nucleotide non-coding RNAs which regulate post-transcription gene expression. Although useful PD biomarkers they have a high potential because of their ability to cross the BBB miRNAs. ${ }^{27}$

\section{Metabolic profiling}

Metabolic profiling of human tissues and/or biological fluids is a reflection of the complex interaction between an individual's genes, proteins and environment. Proton $(1 \mathrm{H})$ and phosphorus (31P) magnetic resonance spectroscopy (MRS) are non-invasive imaging techniques used in the study of energy metabolism metabolites, including ATP, lactate, creatine and other low weight molecular metabolites. Significant metabolite changes have been described in patients with PD. ${ }^{28}$

\section{Neuroimaging biomarkers}

\section{Transcranial B-Mode Sonography (TCS)}

Transcranial B-mode sonography measures the blood flow rate of brain vessels by measuring the ultrasound wave frequency and its echoes. This economical and reliable approach demonstrates the higher echogenicity of substantia nigra(SN) in PD brains that may occur due to increased levels of iron and gliosis in SN with PD. ${ }^{29}$

\section{Magnetic Resonance Imaging (MRI)}

Diffusion weighted imagery is a type of MRI that measures the rate of water diffusion through a tissue to determine the tissue's structural information. The higher calculated diffusivity indicates the greater mobility of water molecules, which can be attributed to cell death and area volume reduction. More traditional MRI techniques, such as highresolution 3-Tesla T1-weighted MRI, can also detect the decreased caudate and putamen volume in patients with PD. ${ }^{30}$

\section{Single-Photon Emission Computed Tomography (SPECT) Scan}

Radionuclide imaging modalities such as PET and SPECT have become the best approach for assessing the metabolism and deficiency of dopamine, using a dopamine transporter ligand. Tracer uptake in the posterior or dorsal striatum is maximally reduced and is asymmetric in Parkinson's disease. $^{31}$

\section{Positron Emission Tomography (PET) Scan}

The PET radiotracer scans emit electron anti-particles (positrons), which are charged positively with the same mass as an electron. The involvement of presynaptic DAT in striatum dopaminergic neurons and $\mathrm{SN}$ can be measured with radiolabeled dopamine analogs ${ }^{18} \mathrm{~F}$ and/or ${ }^{11} \mathrm{C}$. For $\mathrm{PD}$ patients the PET signal of radiolabeled dihydrotetrabenazine DTBZ is lower due to dopaminergic cell loss and subsequent loss of VMAT2.32

\section{Management of PD \\ Levodopa-Carbidopa:}

Dopamine itself cannot cross the blood-brain barrier. Levodopa, an amino acid gets metabolized to form dopamine, which compensates for the dopamine deficiency seen in PD. Small doses of combined carbidopa-levodopa are given in a dose of $25 / 100 \mathrm{mg}$ half tablet twice or thrice a day along with meals. The common adverse effects include nausea, dizziness, and somnolence. 


\section{Dopamine Agonists}

These drugs stimulate dopamine receptors directly. The ergot derivative includes bromocriptine, and the non-ergot derivatives are ropinirole and pramipexole. Pramipexole dosing is $0.125 \mathrm{mg}$ three times a day whereas ropinirole is given as $0.25 \mathrm{mg}$ thrice a day.

\section{Catechol-O-methyltransferase (COMT) inhibitors:}

Entacapone results in blockade of peripheral COMT, an enzyme responsible for the degradation of dopamine. It helps to decrease the breakdown of levodopa, thus increasing its availability to the brain. Entacapone dosing is $200 \mathrm{mg}$ with every dose of levodopa, and up to eight doses can be given each day, whereas the dose of tolcapone is $100 \mathrm{mg}$ three times a day.

\section{Monoamine Oxidase (MAO) Inhibitors}

Drugs like selegiline and rasagiline decrease the metabolism of dopamine by blocking the enzyme, monoamine oxidase. The daily dosing of selegiline is $5 \mathrm{mg}$, usually given in the morning to avoid insomnia. Rasagiline can be initiated with a dose of $0.5 \mathrm{mg}$ once a day and gradually increased to $1 \mathrm{mg}$ once a day.

\section{Amantadine}

It acts by blocking N-methyl-D-aspartate and acetylcholine receptors. It is available as immediate-release tablets or capsules $100 \mathrm{mg}$ each given twice or thrice daily.

\section{Anticholinergic drugs}

Benztropine and trihexyphenidyl result in blockade of acetylcholine receptors. They can be useful for the treatment of tremors and rigidity. Trihexyphenidyl is usually given in a dose of 0.5 to $1 \mathrm{mg}$ twice in a day that is gradually increased to $2 \mathrm{mg}$ thrice in a day. The benztropine dose is 0.5 to $2 \mathrm{mg}$ twice in a day. ${ }^{33}$

\section{Surgery}

Deep brain stimulation rarely used for PD. This involves sending electrical impulses through a neurostimulator system to certain parts of the brain (usually $\mathrm{SN}$ or globus pallidus, which interacts with the $\mathrm{SN}$ ), a brain implant known as a ' brain pacemaker 'The target area of DBS is usually the subthalamic nucleus (STN). The stimulation of the dorsolateral STN border alongside the surgery can improve its efficiency. ${ }^{34}$

\section{Gene Therapy}

In a recent decade, the advancement of PD gene therapy has made considerable progress. Wide loss of dopaminergic neurons is accompanied by decrease within levels of aromatic amino acid decarboxylase (AADC), which transforms 1-DOPA to dopamine. Following positive preclinical studies, adenoassociated viral vectors carrying human AADC genes have recently delivered to $\mathrm{PD}$ patient's putaminal neurons and subthalamic nucleus. By taking appropriate doses of levodopa, sufficient quantities of dopamine production can be managed using this tool. Another target for gene therapy in PD is glutamic acid decarboxylase (GAD) which facilitates GABA production in the subthalamic nucleus of GABA-ergic neurons. ${ }^{35}$

\section{CONCLUSION}

$\mathrm{PD}$ is a chronic, progressive neurodegenerative disease with both engine and non-motor symptoms. Most of the cause remains unknown. Although there is no cure, there are different treatment options, including medication and surgery. Although Parkinson's own is not fatal, complications of the disease may be serious. Non-motor symptoms manifest in $\mathrm{PD}$ long before motor symptoms, and the early presence of non-motor symptoms provides an opportunity for early diagnosis and early treatment of $\mathrm{PD}$, resulting in benefits for patient quality of life and potential cost savings in treatment.

\section{REFERENCES}

1. Parkinson J. An Essay on the Shaking Palsy. London: Sherwood, Neely, and Jones; 1817. pp. 1-16.

2. Twelves D, Perkins KS, Counsell C. Systematic review of incidence studies of Parkinson's disease. Mov Disord. 2003;18(1):19-31.

3. Driver JA, Logroscino G, Gaziano JM, et al. Incidence and remaining lifetime risk of Parkinson disease in advanced age. Neurology. 2009;72(5):32-38.

4. De Lau LM, Breteler MM. Epidemiology of Parkinson's disease. Lancet Neurol. 2006;5(3):525-535.

5. Gourie-Devi M, Gururaj G, Satishchandra P, Subbakrishna DK Prevalence of neurological disorders in Bangalore, India: a community-based study with a comparison between urban and rural areas. Neuroepidemiology. 2004; 23(6):261-8.

6. Das SK, Biswas A, Roy T, Banerjee TK, Mukherjee CS, Raut DK, et al. A random sample survey for prevalence of major neurological disorders in Kolkata. Indian J Med Res. 2006;124(4):163-72.

7. Razdan S, Kaul RL, Motta A, Kaul S, Bhatt RK. Prevalence and pattern of major neurological disorders in rural Kashmir (India) in 1986. Neuroepidemiology. 1994;13(2):113-9.

8. Bharucha NE, Bharucha EP, Bharucha AE, Bhise AV, Schoenberg BS. Prevalence of Parkinson's disease in the Parsi community of Bombay, India. Arch Neurol. 1988;45(1):1321-3.

9. Ragothaman M, Murgod UA, Gururaj G, Louis ED, Subbakrishna DK, Muthane UB. High occurrence and low recognition of Parkinsonism (and possible PD) in old age homes in Bangalore, South India. J Assoc Physicians India. 2008;56(3):233-6

10. DeMaagd G, Philip A. Parkinson's Disease and Its Management: Part 1: Disease Entity, Risk Factors, Pathophysiology, Clinical Presentation, and Diagnosis. P T. 2015;40(5):504-32.

11. Zhou C, Huang Y, Przedborski S. Oxidative stress in Parkinson's disease: a mechanism of pathogenic and therapeutic significance. Ann NY Acad Sci. 2008;1147(3):93-104.

12. Logroscino G. The role of early-life environmental risk factors in Parkinson disease: what is the evidence? Environ Health Perspect. 2005;113(4):1234-1238.

13. Braak H, Del Tredici K, Rüb U, et al. Stasging of brain pathology related to sporadic Parkinson's disease. Neurobiol Aging 2003; 24(1):197-211

14. Massano J, Bhatia KP Clinical Approach to Parkinson's 
Disease: Features, Diagnosis, and Principles of Management. Cold Spring Harb Perspect Med. 2012; 2(6): a008870.

15. Chaudhuri KR, Schapira AH Non-motor symptoms of Parkinson's disease: dopaminergic pathophysiology and treatment. Lancet Neurol. 2009; 8(1):464-74.

16. Gallagher DA, Lees AJ, Schrag A What are the most important nonmotor symptoms in patients with Parkinson's disease and are we missing them? Mov Disord. 2010; 25(3):2493-500.

17. Zlotnik $\mathrm{Y}$ et al Disorders of the Oral Cavity in Parkinson's Disease and Parkinsonian Syndromes. Volume 2015, Article ID 379482, 6 pages http://dx.doi. org $/ 10.1155 / 2015 / 379482$.

18. Jankovic J. Parkinson's disease: clinical features and diagnosis. J Neurol Neurosurg Psychiatry 2008;79(5):368-76.

19. Parkinson's Disease: National Clinical Guideline for Diagnosis and Management in Primary and Secondary Care.Available from: https://www.ncbi.nlm.nih.gov/ books/NBK48502/

20. Fronczek R., Overeem S., Lee S.Y., Hegeman I. M., van Pelt J., van Duinen S. G., et al. Hypocretin (orexin) loss in Parkinson's disease. Brain 2007;130(6):1577-1585.

21. Shigenaga M., Gimeno C. J., Ames B. N. Urinary 8-hydroxy-2'-deoxyguanosine as a biomarker of in vivo oxidative DNA damage. Proc. Natl. Acad. Sci. U.S.A. 1989;8(3):9697-9701.

22. Bentea E., Verbruggen L., Massie A. The proteasome inhibition model of Parkinson's disease. J. Parkinsons Dis. 2017;7(6): 31-63.

23. Martins-Branco D., Esteves A. R., Santos D., Arduino D. M., Swerdlow R. H., Oliveira C. R. Ubiquitin proteasome system in Parkinson's disease: a keeper or a witness? Exp. Neurol. 2012;238(6): 89-99.

24. Nagai Y., Ueno S., Saeki Y., Soga F., Hirano M., Yanagihara T. Decrease of the D3 dopamine receptor mRNA expression in lymphocytes from patients with Parkinson's disease. Neurology 1996;46(1): 791-795.

25. Hong Z., Shi M., Chung K. A., Quinn J. F., Peskind E. R., Galasko D., et al. DJ-1 and alpha-synuclein in human cerebrospinal fluid as biomarkers of Parkinson's disease. Brain 2010;133(4): 713-726.

26. Wang E. S., Sun Y., GuoJ. G., Gao X.,Hu J.W., Zhou L., et al.Tetranectin and apolipoprotein A-I in cerebrospinal fluid as potential biomarkers for Parkinson's disease. Acta Neurol. Scand. 2010; 122(6):350-359.

27. Dos Santos M. C. T., Barreto-Sanz M. A., Correia B. R. S., Bell R., Widnall C., Perez L. T., et al. miRNA-based signatures in cerebrospinal fluid as potential diagnostic tools for early tage Parkinson's disease. Oncotarget 2018;9(4):17455-17465.

28. Havelund J. F., Heegaard N. H. H., Færgeman N. J. K., Gramsbergen J. B. Biomarker research in Parkinson's disease using metabolite profiling. Metabolites 2017;7(3):E42.

29. Skoloudík D., Jelínková M., Blahuta J., Cermák P., Soukup T., Bártová P., et al. Transcranial sonography of the substantia nigra: digital image analysis. AJNR Am. J. Neuroradiol. 2014;35(2):2273-2278.

30. Chung E. J., Kim E. G., Bae J. S., Eun C. K., Lee K.
S., Oh M. Usefulness of diffusion-weighted MRI for differentiation between Parkinson's disease and Parkinson variant of multiple system atrophy. J. Mov. Disord.2009; 2(5): 64-68.

31. Stoessl AJ, Lehericy S, Strafella AP. Imaging insights into basal ganglia function, Parkinson's disease, and dystonia. Lancet 2014;384(3):532-44.

32. Bohnen N. I., Albin R. L., Koeppe R. A., Wernette K. A., Kilbourn M. R., Minoshima S., et al. Positron emission tomography of monoaminergic vesicular binding in aging and Parkinson disease. J. Cereb. Blood Flow Metab 2006; 26(4): 1198-1212.

33. Shrimanker S, Tadi P, Manso JCS. Parkinsonism. Available from: https:/www.ncbi.nlm.nih.gov/books/ NBK542224/.

34. Herzog J., Fietzek U., Hamel W., Morsnowski A., Steigerwald F., Schrader B. Most effective stimulation site in subthalamic deep brain stimulation for Parkinson's disease. Mov. Disord. 2004;19(4):1050-1054.

35. Mittermeyer G., Christine C. W., Rosenbluth K. H., Baker S. L., Starr P., Larson P., et al. Long-term evaluation of a phase 1 study of AADC gene therapy for Parkinson's disease. Hum. Gene Ther. 2012;23(1): 377-381.

Source of Support: Nil; Conflict of Interest: None

Submitted: 17-01-2020; Accepted: 15-02-2020; Published online: 12-03-2020 